\section{Enfisema orbitario espontáneo tras la maniobra de Valsalva}

\author{
SEBASTIÁN MUÑOZ G. ${ }^{1,2}$, RICARDO RÍOS B. ${ }^{1}$, MANUEL RODRÍGUEZ O. ${ }^{2}$, \\ CARLOS PALMA D. ${ }^{2}$, PRISCILA ZÚÑIGA G. ${ }^{2}$
}

\section{Orbital emphysema after Valsalva maneuver. Report of one case}

Orbital emphysema is a rare complication of orbital and facial trauma, involving the sinuses and occasionally the nasal cavity. Most cases occur as a result of trauma but spontaneous orbital emphysema has been reported. Clinical manifestations are often delayed until the upper airway pressure increases as when blowing or coughing. Along with the fracture, the rupture of the underlying mucosa is essential for emphysema to manifest. We report a 29-years-old male who consulted in the emergency room due to eyelid swelling after a Valsalva maneuver, a couple of hours after suffering a facial injury secondary to a fall. In the presence of eyelid emphysema, the patient underwent craniofacial imaging studies, confirming an ethmoidal lamina papyracea fracture.

(Rev Med Chile 2015; 143: 257-261)

Key words: Ethmoid bone; Facial injuries; Orbital fractures; Subcutaneous emphysema.

\author{
'Hospital Lord Cochrane de la \\ comuna de Cochrane, Servicio de \\ Salud Aysén. Chile. \\ ${ }^{2}$ Hospital Dr. Leopoldo Ortega \\ de Chile Chico, Servicio de Salud \\ Aysén. Chile. \\ Recibido el 31 de marzo de 2014, \\ aceptado el 5 de diciembre de \\ 2014. \\ Correspondencia a: \\ Sebastián Muñoz G. \\ Médico Cirujano en Etapa de \\ Destinación y Formación M.Sc \\ Hospital Dr. Leopoldo Ortega de \\ Chile Chico. Chile. \\ Calle Caupolicán sin número. \\ Comuna de Chile Chico. \\ Teléfono +56-9-76964414 \\ sebastiangroff@gmail.com
}

1 1 enfisema orbitario (EO) es una complicación potencial de las fracturas de la pared orbitaria medial. La mayoría de los casos ocurren por trauma ${ }^{1}$. Sin embargo, se han reportado casos en que el EO sucedería espontáneamente, secundario a acciones que impliquen un aumento de la presión en la vía aérea superior, como estornudar o sonarse la nariz, en cuyos casos se presume pudiera existir alguna malformación en la zona ${ }^{2}$. En el EO se produce paso de aire desde las fosas nasales y/o senos paranasales a la órbita y al tejido subcutáneo. Las manifestaciones pueden retrasarse algún tiempo hasta que se presente el momento de aumento la presión dentro de la vía aérea superior.

\section{Caso clínico}

Paciente masculino de 29 años que consultó de urgencia por presentar aumento de volumen palpebral izquierdo tras sonarse la nariz (maniobra de Valsalva). Esa misma madrugada había sufrido una caída de altura golpeándose en la cara lateral izquierda de la nariz, a la altura de los huesos propios, tras lo cual presentó epistaxis leve y autolimitada. El examen oftalmológico reveló visión 20/20 en cada ojo, reflejos pupilares normales y motilidad ocular conservada, sin edema ni hiperemia conjuntival, sólo discreta equimosis en la región medial de la hemicara izquierda acompañada de aumento de volumen palpebral crepitante a la palpación. El marco óseo orbitario y la pirámide nasal estaban sin dolor. Los signos vitales eran normales. El estudio radiológico cráneo-facial corroboró la presencia de enfisema subcutáneo y orbitario, asociado a fractura de la lámina papirácea del etmoides ipsilateral. El paciente fue hospitalizado y observado por $24 \mathrm{~h}$, posteriormente fue dado de alta con la precaución de evitar maniobras que pudieran aumentar la presión de la vía aérea superior, durante dos semanas. El enfisema se resolvió espontáneamente dentro de 5 días y durante las dos semanas siguientes el paciente no desarrolló complicaciones (Figuras 1 a 3 ). 

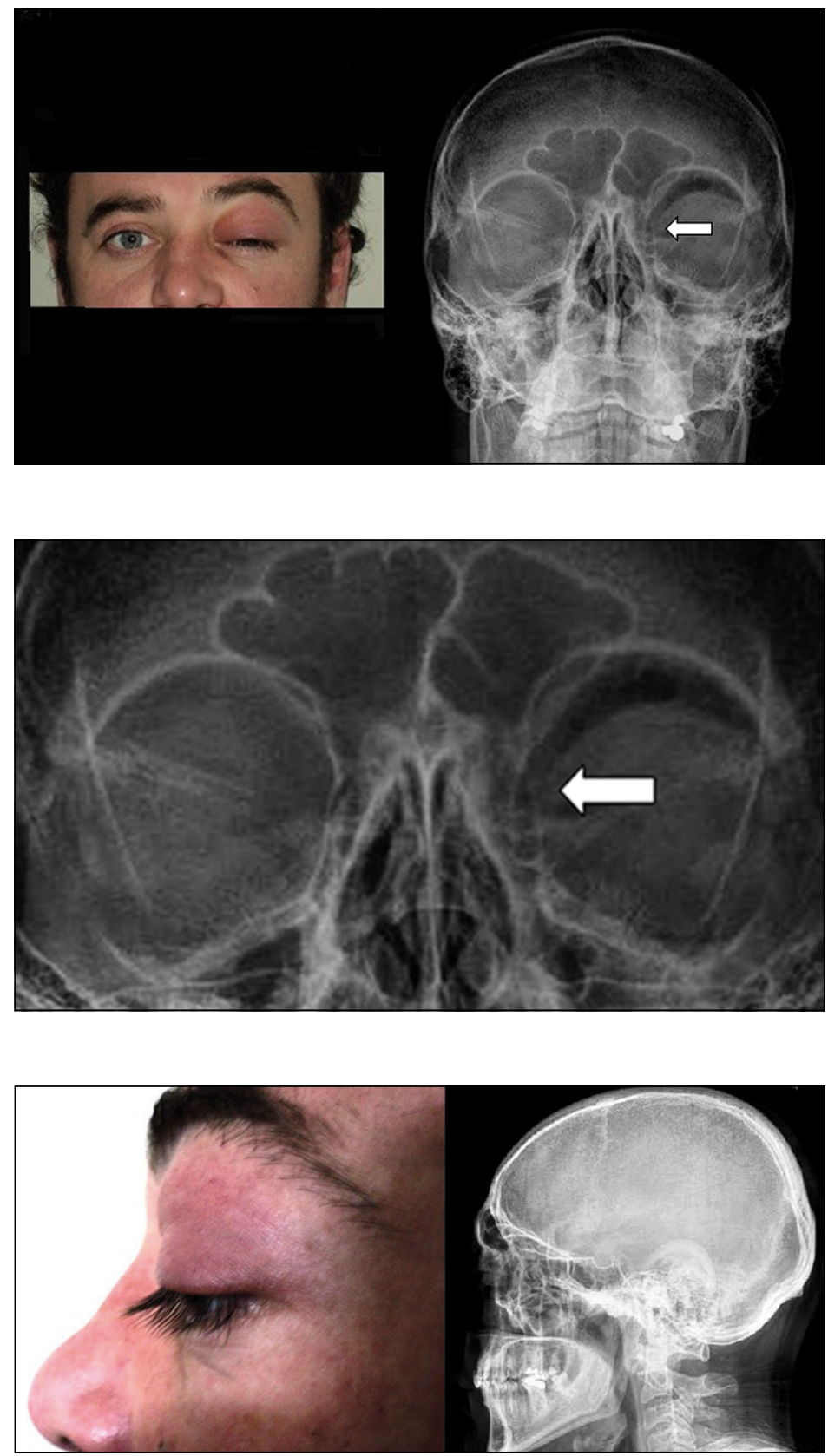

Figura 1. A la izquierda se observa el rostro del paciente en que se aprecia el aumento de volumen palpebral acompañado de equimosis del canto medial del ojo izquierdo y de parte de la cara lateral izquierda de la región nasal que colinda con el ojo del lado izquierdo. A la derecha, se observa una imagen radiográfica posteroanterior de cráneo del mismo paciente en que se aprecia bajo el arco ciliar izquierdo la presencia de aire, además se observa la solución de continuidad de la lámina papirácea izquierda (flecha).

Figura 2. Selección aumentada de la radiografía posteroanterior en que se aprecia con mayor claridad la solución de continuidad de la lámina papirácea del lado izquierdo (flecha).

Figura 3. A la izquierda se observa imagen lateral del globo ocular del paciente con marcado aumento de volumen palpebral. A la derecha se observa la imagen radiográfica lateral de cráneo en que se aprecia el enfisema palpebral.

\section{Discusión}

El EO es una entidad poco frecuente que consiste en el paso de aire de las fosas nasales y/o senos paranasales al tejido subcutáneo y a la órbita; la mayoría se origina como consecuencia de un traumatismo orbitario y/o facial ocasionando algún tipo de fractura ${ }^{3}$. Dentro de este contexto, pese a que la pared medial de la órbita supone ser la más débil, las fracturas del complejo nasoetmoidal son las menos frecuentes de las fracturas faciales. Adicionalmente, existen casos descritos 
en que el enfisema ha ocurrido a consecuencia de iatrogenia, por infección local o bien de manera espontánea sin una causa aparente, en cuyo caso un barotrauma produciría una microfractura de la lámina papirácea y el consecuente paso de aire desde los senos etmoidales hacia la cavidad orbitaria y tejido subcutáneo. En una serie de 129 pacientes se describe la fractura de la pared medial de la órbita como la causa más común de EO. La edad promedio de presentación para esta patología correspondió a los 33 años y tuvo una relación de incidencia hombre/mujer de 7 a $1^{4}$ (Tabla 1).

La entrada de aire en la cavidad orbitaria no suele ser contemporánea con el momento de la lesión, sino cuando ocurre aumento de la presión dentro de la vía respiratoria superior de manera artificial$^{2}$. En la mayoría de los casos la condición es benigna y autolimitada, pero en algunas ocasiones el edema orbitario puede evolucionar tórpidamente con síntomas visuales secundarios a isquemia de la arteria central de la retina provocada por un síndrome compartimental en la cavidad orbitaria, con compromiso del nervio óptico y pérdida de la visión ${ }^{5}$. Según el sitio donde se encuentre alojado el aire en la cavidad orbitaria, podemos encontrar distintos tipos de $\mathrm{EO}^{2}$ :

- EO subperióstico: el aire entra a la órbita subperiósticamente y las estructuras periorbitarias permanecen intactas ${ }^{2}$.

- EO retroseptal: el aire ingresa a la órbita por detrás del septum orbitario y se acumula en la órbita empujando el globo ocular hacia adelante. Característicamente el aire se acumula

\section{Tabla 1. Relación de causalidad del enfisema orbitario}

\begin{tabular}{|lc|}
\hline Causas de enfisema orbitario & Frecuencia \\
\hline Fractura pared medial de la órbita & $40 \%$ \\
Fractura del suelo orbitario por estallido & $34 \%$ \\
Fractura malar & $11 \%$ \\
Fractura seno paranasal & $8 \%$ \\
$\begin{array}{l}\text { Fractura pared medial asociada a fractura } \\
\text { piso órbita }\end{array}$ & $6 \%$ \\
$\begin{array}{l}\text { Enfisema espontáneo sin relación } \\
\text { traumática }\end{array}$ & $1 \%$ \\
\hline
\end{tabular}

Lloyd G. Radiology of the Orbit. Philadelphia, Saunders. 1975. en la porción superior de la órbita, tras el septo orbitario. El sitio del paso del aire suele ser la lámina papirácea del etmoides, pero en raras ocasiones hay comunicación entre los senos frontales o esfenoidales y la órbita. Los hallazgos clínicos de esta variedad son similares al hematoma subperióstico, en el que hay evidencia de incremento de la tensión intra-orbitaria con proptosis, pérdida del pliegue de belleza, y ocasionalmente diplopia ${ }^{2}$.

- EO preseptal: de muy rara ocurrencia; consiste en la presencia de aire en los párpados; se origina por fractura de los huesos lacrimales los cuales se encuentran anterior al sitio de unión del septum orbitario. El aire ingresa directamente al tejido palpebral desde la cavidad nasal ${ }^{2}$.

- Combinación de EO retroseptal y preseptal: en este caso el EO retroseptal atraviesa el septo orbitario e invade los tejidos preseptales. Para esto la presión intraorbitaria debe ser lo suficientemente alta para atravesar el septo ${ }^{2}$.

Al momento de evaluar a un paciente con aumento de volumen orbitario es indispensable realizar una exhaustiva exploración física regional. Ésta debe incluir: inspección y palpación de la región afectada, valoración de la movilidad ocular, agudeza visual y el estado del reflejo pupilar aferente. La presencia de crepitación de la región afectada hace sospechar la ocurrencia del EO en cuyo caso, la motilidad ocular puede verse limitada por compromiso muscular, cuando la presión dentro de la cavidad orbitaria se encuentra muy elevada ${ }^{6}$. Las manifestaciones clínicas pueden variar desde un simple aumento de volumen palpebral hasta la diplopia, dolor ocular o pérdida completa de la visión. En las fracturas de la pared interna suele aparecer equimosis y epistaxis ${ }^{6}$. Se debe tener presente que en los casos de enfisema retroseptal ocurrirá proptosis y la crepitación de la cavidad orbitaria sólo será palpable indirectamente al ejercer presión sobre el globo ocular por encontrarse el aire alojado tras el septo orbitario.

El estudio radiográfico permite demostrar la presencia de aire en la cavidad orbitaria. El enfisema palpebral suele presentarse como una pequeña área radiolúcida en la región media de la órbita en la proyección frontal, el cual se encuentra separado del enfisema retroseptal, pues este último tiende 
a localizarse en la porción superior de la órbita. La radiología convencional permite diagnosticar la presencia de enfisema y localizar la fractura en aproximadamente el $70 \%$ de casos, siendo necesario recurrir a la tomografía computarizada de órbita en los casos no diagnosticados mediante radiología simple o cuando se requiera cuantificar el enfisema. Toda vez que se produzca un traumatismo orbitario es recomendable solicitar una radiografía de órbita antero-posterior, lateral y de Waters, que suele ser la proyección más apropiada para demostrar la presencia de fracturas en las paredes orbitarias.

La mayoría de casos de EO son autolimitados y no requieren tratamiento específico para su resolución ${ }^{2,7,8}$. No existe un claro consenso que la administración de tratamientos descongestionantes y humidificantes nasales faciliten la vasoconstricción de la mucosa de los senos y el drenaje del aire. Puesto que la fibrosis alrededor de la lesión en la lámina papirácea se espera ocurra dentro de dos semanas y, con el fin de evitar que se produzca una solución de continuidad permanente entre las cavidades orbitaria y paranasal, todo paciente con EO debe evitar las conductas que provoquen la maniobra de Valsalva (esfuerzos físicos, sonarse, etc.) $)^{3,9}$. La profilaxis antibiótica está indicada en los casos de lesiones contaminadas, antecedente de sinusitis o inmunosupresión. Los casos graves, en que se presentan síntomas visuales, pueden requerir la evacuación urgente del aire de la cavidad orbitaria, la cual puede realizarse mediante punciones-aspiraciones con un catéter venoso 24 G o por cantotomía lateral y cantolisis, dependiendo de la intensidad de las manifestaciones y lo familiarizado que esté el médico tratante con el procedimiento. En rigor, este nivel de intervención debe ser practicado por especialista oftalmólogo. Las indicaciones de cantotomía lateral incluyen la presencia de hematoma retrobulbar con incremento de la presión intraocular (> $40 \mathrm{mmHg}$ ), defectos en el reflejo pupilar aferente, disminución de la visión, proptosis, y disminución de la motilidad ocular ${ }^{10}$. Pese a que la cantotomía lateral puede ser una intervención eficaz, no se encuentra exenta de riesgos y puede resultar en daño periocular significativo como ectropión, ptosis, daño en la glándula lacrimal o su arteria, daño en el globo ocular o daño estético. Los casos en que exista un trauma ocular abierto la cantotomía lateral se encuentra contraindicada ${ }^{8,11}$.

\section{Conclusión}

La presencia de epistaxis como consecuencia de un traumatismo facial seguida de enfisema orbitario que se desarrolla tras la realización de la maniobra de Valsalva, aún en ausencia de equimosis y/o dolor a la exploración de los rebordes óseos y con normalidad radiológica, es patognomónica de lesión de la lámina papirácea del etmoides. Dadas las potenciales consecuencias deletéreas que pueden acarrear los casos graves, resulta imprescindible realizar una minuciosa exploración física e imagenológica para hacer el diagnóstico y descartar la ocurrencia de posibles complicaciones, circunstancias que podrían indicar la necesidad de corrección quirúrgica. Tomando en cuenta estas consideraciones, los médicos que trabajan en los Servicios de Urgencia debieran estar familiarizados con las formas de descompresión quirúrgica de la cavidad orbitaria, conociendo sus riesgos y beneficios y de su oportuna derivación si las circunstancias lo permiten.

\section{Referencias}

1. Crossrow JI, Curtis JA, Edeiken J. Enophthalmos Simulating Orbital Emphysema. Roentgenol 1977; 129: 728-9.

2. Brady FA, Roser SM, Hieshima GB. Orbital Emphysema. British Journal of Oral Surgery 1976; 14: 65-71.

3. Casarramona Lobera F, Belda Jornet V, Cruz Llobet J, Pou Calvo R, Masip Español M, E. Coma Salvans E. Enfisema palpebral espontáneo. Emergencias 2005; 17: 148-9.

4. Birrer RB, Robinson T, Papachristos P. Orbital emphysema: how common, how significant? Ann Emerg Med 1994; 24 (6): 1115-8.

5. Nassr MA, Morris CL, Netland PA, Karcioglu ZA. Intraocular Pressure Change in Orbital Disease. Surv Ophthalmol 2009; 54 (5): 519-44.

6. Casarramona Lobera F, Belda Jornet V, Pou Calvo R, Cruz Llobet J, Daza López M, Cuadrada Majó CE. Enfisema palpebral por fractura de la lámina papirácea del etmoides. emergencias 2003; 15: 247-9.

7. Conor O'Malley CL. Orbital Emphysema simulating cellulitis. Br J Ophthalmol. 1943; 27 (5): 222-6.

8. Hunts JH, Patrinely JR, Holds JB, Anderson RL. Orbital emphysema. Staging and acute management. Ophthalmology 1994; 101 (5): 960-6.

9. Taguchi Y, Sakakibara Y, Uchida K, Kishi H. Orbital emphysema following nose blowing as a sequel of a snowboard related head injury. Br J Sports Med 2004; 
Enfisema orbitario espontáneo - S. Muñoz et al

38: e28.

10. Weber D, Shaw S, Winslow J. Images in Emergency Medicine. Ann Emerg Med. 2009; 54: 635.

11. McInnes G, Howes DW. Lateral canthotomy and cantholysis: a simple, vision saving procedure. CJEM. 2002; 4: 49-52.

12. Lloyd G. Radiology of the Orbit. Philadelphia, Saunders. 1975.
13. Li T, Mafee MF, Edward DP. Bilateral Orbital Emphysema From Compressed Air Injury. Am J Ophthalmol 1999; 128: 103-4.

14. Kaplan K, Winchell GD. Orbital emphysema from nose blowing. N Engl J Med 1968; 278: 1234.

15. Ridvan Alimehmeti, Artan Gjika, Jera Kruja. Orbital emphysema after nose blowing. Neurology 2011; 76; 1274 . 